\title{
Intelligent Base Station Association for UAV Cellular Users: A Supervised Learning Approach
}

\author{
Boris Galkin*, Ramy Amer*, Erika Fonseca*, and Luiz A. DaSilva*† \\ * CONNECT- Trinity College Dublin, Ireland \\ $\dagger$ Commonwealth Cyber Initiative, Virginia Tech, USA \\ E-mail: \{galkinb,ramyr,fonsecae\}@tcd.ie,ldasilva@vt.edu
}

\begin{abstract}
Fifth Generation (5G) cellular networks are expected to provide cellular connectivity for vehicular users, including Unmanned Aerial Vehicles (UAVs). When flying in the air, these users experience strong, unobstructed channel conditions to a large number of Base Stations (BSs) on the ground. This creates very strong interference conditions for the UAV users, while at the same time offering them a large number of BSs to potentially associate with for cellular service. Therefore, to maximise the performance of the UAV-BS wireless link, the UAV user needs to be able to choose which BSs to connect to, based on the observed environmental conditions. This paper proposes a supervised learning-based association scheme, using which a UAV can intelligently associate with the most appropriate BS. We train a Neural Network (NN) to identify the most suitable BS from several candidate BSs, based on the received signal powers from the BSs, known distances to the BSs, as well as the known locations of potential interferers. We then compare the performance of the $\mathrm{NN}$-based association scheme against strongest-signal and closest-neighbour association schemes, and demonstrate that the NN scheme significantly outperforms the simple heuristic schemes.
\end{abstract}

Index Terms-Cellular-connected UAVs, Machine Learning, Supervised Learning.

\section{INTRODUCTION}

$5 \mathrm{G}$ cellular networks are intended to meet the strict latency and reliability requirements of Ultra-Reliable Low Latency Communication (URLLC), thereby enabling a wide range of new technologies and use-cases. Autonomous vehicles such as self-driving cars and UAVs are predicted to be some of the core users of $5 \mathrm{G}$ networks [1]. UAVs are becoming increasingly used in a wide range of applications such as aerial surveillance, safety, as well as product delivery [2]. In all of these use-cases, the UAVs require ubiquitous and reliable data communication with their human operators, local authorities, as well as each other, which makes them reliant on the $5 \mathrm{G}$ network.

These UAV users represent a paradigm shift for the cellular network, as they are able to freely move in three-dimensional space, unlike typical terrestrial users. As they move in the air, UAVs are exposed to vastly different radio environment conditions as compared to terrestrial users, due to the presence of dominant Line-of-Sight (LoS) links as well as reduced antenna gains from BS down-tilted antennas [3]. For instance, UAVs can establish unobstructed LoS wireless links to distant transmitters, which allows them to receive a strong, unatten- uated wireless signal from their serving BS, but which also make them susceptible to strong LoS interference.

Recent $3^{\text {rd }}$ Generation Partnership Program (3GPP) studies on the performance of cellular connectivity for UAV users confirm that strong interference in the sky will have a severe impact on UAV users [4]. The studies suggest that this interference can be mitigated by equipping the UAVs with steerable, directional antennas. By steering such an antenna towards the desired serving BS, the UAV can use the strong directional antenna gain to boost the desired signal, while simultaneously attenuating undesirable interfering signals. In our prior work [5], we mathematically model the achievable performance of a UAV equipped with a steerable antenna which connects to terrestrial infrastructure. Our results demonstrate that UAVs operating at large heights above ground experience strong interference from a large number of BSs with LoS channel conditions, which can be compensated for via steerable directional antennas, corroborating the conclusions in [4]. In [6], we investigate the handover of UAVs under practical antenna configurations. The authors in [7] examine how factors such as BS density and height above ground affect the ability of UAVs and ground users to share the network. The same authors in [8] extend this by studying the impact of directional antenna tilt and beamwidth. In [9] the authors model the performance of a UAV with an omni-directional antenna connecting to a BS network with Multiple Input Multiple Output (MIMO) antenna arrays, and demonstrate how multi-user MIMO can significantly improve UAV service quality.

The current state-of-the-art on UAV cellular communications tends to assume that the UAV has full awareness of the environment and is therefore able to associate with the most suitable BS. Given its aerial position, a UAV may have a large number of candidate BSs that it can connect to for cellular service, using its directional antenna. To choose the most suitable BS the UAV needs to be aware of the channel conditions for each candidate BS, which involves steering the directional antenna towards each BS and assessing the resulting channel quality. Depending on the UAV use case and the state of the environment, this process may introduce a large overhead to maintaining cellular connectivity, or (in the case of highly dynamic channels when the UAV is moving) it may not be feasible at all. As an alternative to this iterative channel measurement step, we propose choosing the most suitable 
candidate BS using available environmental knowledge and a trained NN.

NNs have started to gain popularity in the wireless community as function approximators [10]. In this regard, the authors in [11] have explored the use of supervised learning for training millimeter-wave MIMO antennas. The authors demonstrate how the observed channel conditions at one antenna can be used to configure an antenna at another location, using a trained NN. In [12], the authors use BS geolocation information to design an NN-based scheduler that maximises the system throughput in a millimeter-wave multiBS, multi-user communication scenario. The work in [13] proposes NN-based coordinated beamforming, where multiple BSs simultaneously serve a single user.

Our contribution in this paper is to propose an NN approach which allows a UAV user to intelligently select one of the BSs in the network to associate with, so that the channel quality of the UAV-BS link is maximised. We consider a UAV equipped with two sets of RF-chains with separate antennas: an omni-directional antenna for measuring the received signal power from nearby BSs, as well as a directional antenna which the UAV aligns towards its associated BS and uses for data transmission. We train an $\mathrm{NN}$ to infer which $\mathrm{BS}$ will give the best channel quality for the directional antenna connection based on the received signal power at the omni-directional antenna, as well as other environmental information that is known to the UAV. The UAV is capable of using heuristic BS selection strategies; to demonstrate the advantage of our $\mathrm{NN}$ approach we compare the results against these heuristic strategies, based on both simulations as well as mathematical derivations from our prior works [5] and [6].

\section{SySTEM MODEL}

We consider an urban environment where a flying UAV uses an underlying cellular network for its wireless connectivity, as depicted in fig. 1. The underlying cellular network consists of BSs which are horizontally distributed as a homogeneous Poisson point process (PPP) $\Phi=\left\{x_{1}, x_{2}, \ldots\right\} \subset \mathbb{R}^{2}$ of intensity $\lambda$, at a height $\gamma_{G}$ above ground. Elements $x_{i} \in \mathbb{R}^{2}$ represent the projections of the BS locations onto the $\mathbb{R}^{2}$ plane. The coordinates of the UAV are denoted as $x_{0} \in \mathbb{R}^{2}$, with the UAV height above ground denoted as $\gamma$. Let $r_{i}=\left\|x_{i}-x_{0}\right\|$ denote the horizontal distance between the coordinates $x_{0}$ and $x_{i}$, and let $\phi_{i}=\arctan \left(\Delta \gamma / r_{i}\right)$ denote the vertical angle, where $\Delta \gamma=\gamma-\gamma_{G}$. Without loss of generality we set the horizontal coordinates of the UAV $x_{0}$ as the origin $(0,0)$.

The UAV is equipped with two sets of antennas: an omnidirectional antenna for BS pilot signal detection and signal strength measurement, as well as a directional antenna for communicating with the UAV's associated BS. The omnidirectional antenna has an omni-directional radiation pattern with an antenna gain of 1 , while the directional antenna has a horizontal and vertical beamwidth $\omega$ and a rectangular radiation pattern; following [5], the antenna gain is given as $\eta(\omega)=16 \pi /\left(\omega^{2}\right)$ inside the main lobe and $\eta(\omega)=0$ outside. We denote the coordinates of the BS which the UAV
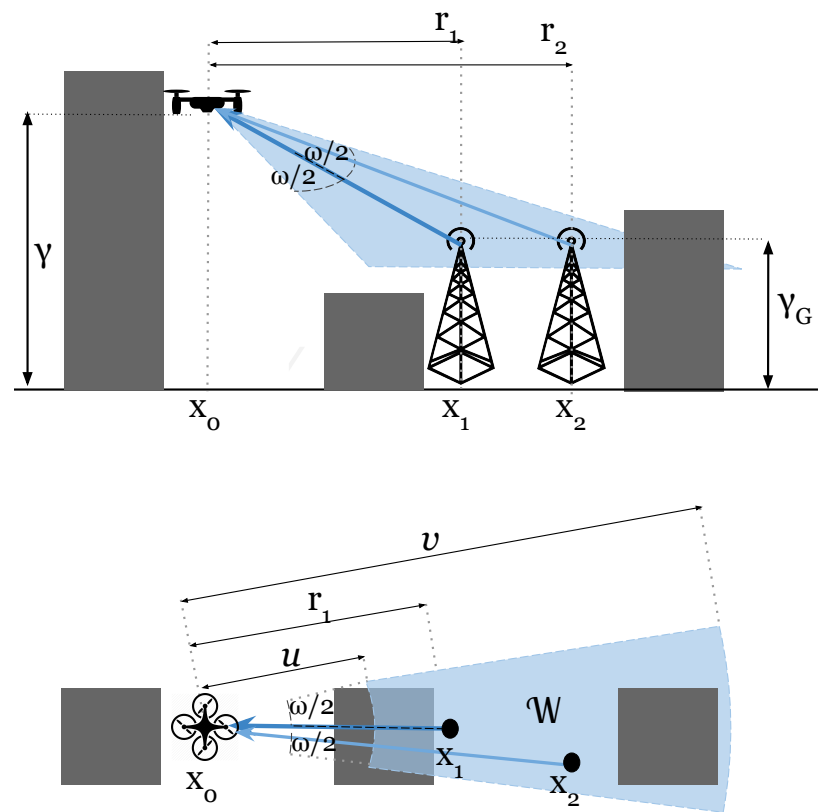

Fig. 1. Side and top view showing a UAV in an urban environment at a height $\gamma$, positioned above $x_{0}$ with antenna beamwidth $\omega$. The UAV associates with the BS at $x_{1}$ and centers its antenna main lobe on the BS location; the blue area $\mathcal{W}$ illuminated by the main lobe denotes the region where interferers may be found. The BS at $x_{2}$ falls inside this area and produces interference.

is associated with as $x_{s} \in \Phi$ and its horizontal distance to the UAV as $r_{s}$. The UAV aligns its directional antenna towards $x_{s}$; this results in the formation of an antenna radiation pattern around $x_{s}$ which we denote as $\mathcal{W} \subset \mathbb{R}^{2}$, as depicted in fig. 1. This area takes the shape of a ring sector of arc angle equal to $\omega$ and major and minor radii $v\left(\gamma, r_{s}\right)$ and $u\left(\gamma, r_{s}\right)$, respectively, where

$$
\begin{aligned}
& v\left(\gamma, r_{s}\right)= \begin{cases}\frac{|\Delta \gamma|}{\tan \left(\left|\phi_{s}\right|-\omega / 2\right)} & \text { if } \omega / 2<\left|\phi_{s}\right|<\pi / 2-\omega / 2 \\
\frac{|\Delta \gamma|}{\tan (\pi / 2-\omega)} & \text { if }\left|\phi_{s}\right|>\pi / 2-\omega / 2 \\
\infty & \text { otherwise }\end{cases} \\
& u\left(\gamma, r_{s}\right)= \begin{cases}\frac{|\Delta \gamma|}{\tan \left(\left|\phi_{s}\right|+\omega / 2\right)} & \text { if }\left|\phi_{s}\right|<\pi / 2-\omega / 2 \\
0 & \text { otherwise }\end{cases}
\end{aligned}
$$

with |.| denoting absolute value. The BSs which fall inside the area $\mathcal{W}$ are denoted by the set $\Phi_{\mathcal{W}}=\{x \in \Phi: x \in \mathcal{W}\}$. The BSs in the $\Phi_{\mathcal{W}}$ are capable of causing interference to the UAV-BS communication link, as their signals may be received by the UAV's directional antenna with non-zero gain.

In our scenario we consider an urban environment, with a grid of buildings distributed according to a square grid, following the model proposed in [14]. All of the buildings are modelled as having the same square horizontal area, with each building having a Rayleigh-distributed random height. These buildings are capable of blocking the wireless link between a BS and the UAV. To determine if the channel between the UAV and a BS is LoS or non-Line-of-Sight (NLoS) we carry 
out a ray-trace; if a building exists between the UAV and BS that is tall enough to block the straight line between the two devices then the channel is considered NLoS.

We assume that the BSs are equipped with Uniform Linear Array (ULA) antennas, with $N_{t}$ antenna elements. The vertical gain of these antennas is a function of the angle between the $\mathrm{UAV}$ and the BS and is defined similar to [15] as

$$
\mu\left(\phi_{i}\right)=\frac{1}{N_{t}} \frac{\sin ^{2} \frac{N_{t} \pi}{2} \sin \left(\phi_{i}\right)}{\sin ^{2} \frac{\pi}{2} \sin \left(\phi_{i}\right)} .
$$

For simplicity we consider the BS horizontal gain to be omni-directional with a value of 1 .

When the UAV is connected to the $\mathrm{BS}$ at $x_{s}$ and aligns its directional antenna towards it, the Signal-to-Interference-andNoise Ratio (SINR) of the downlink signal received by the directional antenna is given as

$$
\mathrm{SINR}=\frac{p H_{t_{s}} \eta(\omega) \mu\left(\phi_{s}\right) c\left(r_{s}^{2}+\Delta \gamma^{2}\right)^{-\alpha_{t_{s}} / 2}}{I_{L}+I_{N}+\sigma^{2}}
$$

where $p$ is the $\mathrm{BS}$ transmit power, $H_{t_{s}}$ is the random Nakagami-m multipath fading component, $\alpha_{t_{s}}$ is the pathloss exponent, $t_{s} \in\{\mathrm{L}, \mathrm{N}\}$ is an indicator variable which denotes whether the UAV has LoS or NLoS to its serving BS $x_{s}, c$ is the near-field pathloss, $\sigma^{2}$ is the noise power, and $I_{L}$ and $I_{N}$ are the aggregate interference from the BSs which have LoS and NLoS channels to the UAV, respectively.

We define an SINR threshold $\theta$ for the wireless link: if SINR $>\theta$ this represents the UAV establishing a wireless link to the BS at $x_{s}$ of an acceptable channel quality. We refer to this as the UAV having coverage from the cellular network.

We assume that the UAV has the 3D coordinates of the BS network $\Phi$, either from a map supplied by the network operator, or through sensing by the UAV itself. Using this information, in addition to measurements received by the UAV's omni-directional antenna, the UAV makes a decision about which BS in $\Phi$ it should associate with, for the purpose of maximising the SINR of its communication link. In the next section we describe our proposed supervised learning-based $\mathrm{NN}$ architecture to carry out this process.

\section{MACHINE LEARNING APPROACH}

\section{A. Neural Network Architecture and Configuration}

The architecture of an NN model includes the number of layers, number of neurons per layer, and how these neurons are connected. This architecture determines how complex it will be to calculate the optimal values for a specific task. A $\mathrm{NN}$ with more layers and neurons typically requires a larger dataset for training. Our NN is composed of the input layer, two hidden layers, and one output layer, as depicted in fig. 2 .

For NN approaches, it is essential to define which features of the environment will be relevant to an effective solution. We use these features as the inputs of our model so that it may accurately react to the conditions of the environment. Our objective for the NN model is to have it identify which of the BSs in $\Phi$ will provide the highest SINR when connected via the directional antenna. This corresponds to a classification problem, wherein the $\mathrm{NN}$ is trained to choose from one of several discrete options, given a provided input. Let $\Phi_{\zeta} \subset \Phi$ denote the $\zeta$ closest BSs to the UAV; the NN will choose the serving BS from within this set, based on which BS it believes to have the highest directional antenna SINR.

The NN takes several measurements relating to the BSs in $\Phi_{\zeta}$ as inputs. First, it takes the time-averaged received signal powers from each of the relevant BSs, as measured by the omni-directional antenna $\mathbf{P}_{\zeta}=\left\{p_{1}, p_{2}, \ldots, p_{\zeta}\right\}$, where $p_{i}=p \mu\left(\phi_{i}\right)\left(r_{i}^{2}+\Delta \gamma^{2}\right)^{-\alpha_{t_{i}} / 2}$. The signal is time-averaged to remove the multipath fading effects. As the UAV has access to the position information of the BSs, the NN also takes the horizontal distances $\mathbf{R}_{\zeta}=\left\{r_{1}, r_{2}, \ldots, r_{\zeta}\right\}$ to the BSs in $\Phi_{\zeta}$ as inputs.

Using this same position information, along with knowledge of its directional antenna, the NN only accounts for interfering BSs within its directional antenna beamwidth by taking in the horizontal distances only to these BSs. We denote a $\zeta \times$ $\xi$ matrix as $\mathbf{F}_{\zeta}$, where each row corresponds to one of the candidate BSs, and each column corresponds to one of the $\xi$ closest BSs that would cause interference for the UAV if it attempted to communicate with one of the candidate BSs. In other words, $\mathbf{F}_{\zeta}(i, j)$ represents the distance from the UAV to the $j$-th closest BS belonging to $\Phi_{\mathcal{W}_{i}}$, which is the set of BSs within the area $\mathcal{W}_{i}$ illuminated by the UAV directional antenna when aligning towards BS $i$. In the event that $\Phi_{\mathcal{W}_{i}}$ contains fewer than $\xi \mathrm{BSs}$, the remaining entries in the $i$-th row of $\mathbf{F}_{\zeta}$ are set to null values.

Finally, the UAV takes it own height above ground $\gamma$ as an input into the NN.

The NN training itself requires the fine-tuning of parameters related to the learning rate and convergence of the classification, known as the hyperparameters. Hyperparameters are parameters chosen before the training process that can improve the learning process.

We detail our choices for the hyperparameters below:

- Learning Rate: is the amount by which the weights in an NN model are updated. We set it to $10^{-5}$; with this value, the model does not overfit to our training data.

- Epoch: is an iteration of the training process where the model is filled with all the elements of the training dataset. If a model is trained with too many epochs, it can overfit to the training data, while if a model uses too few epochs, it might not learn the necessary features to perform the classification. After testing several values, we set the number of epochs to 200 .

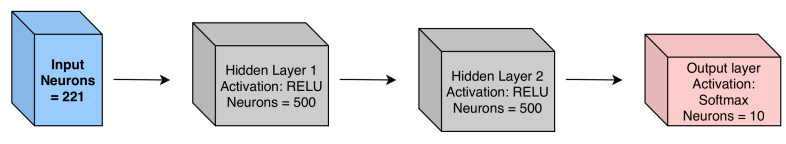

Fig. 2. Architecture of the proposed NN, with the number of input neurons corresponding to $\zeta=10, \xi=20$ 
- Optimiser: is the function that modifies the weights of each neuron with the purpose of minimising the loss function. The loss function indicates how close the output of the model is to the expected result. The main objective of the learning process is to optimise the loss function, making the predicted output closer to the expected one without over-fitting to the training data. We choose the optimiser AdaMax because it has the feature of accelerating the search for the minimum value of the loss function and reducing oscillations. In addition, it is less sensitive to the choice of the hyper-parameters when compared to the Adaptive Moment Optimisation (Adam) optimizer.

\section{B. Simulation \& Training}

In supervised learning, for a model to learn it must first be trained with a set of labelled data, and then tested with a second set to evaluate its accuracy. To avoid overfitting the model this second dataset cannot be used in the training process itself. To generate our datasets, we simulate the environment described in the System Model section, with a random PPP distribution of BSs $\Phi$, random building heights, and the UAV at $x_{0}$ at a random height $\gamma$. The simulation is carried out in the $\mathrm{R}$ statistical language, using the $\mathrm{R}$ Keras library [16] for the $\mathrm{NN}$ architecture. We record the values of $\mathbf{P}_{\zeta}, \mathbf{R}_{\zeta}$, and $\mathbf{F}_{\zeta}$, as observed by the UAV. We then have the UAV iteratively align its directional antenna with each of the candidate BSs in $\Phi_{\zeta}$ and we measure the time-averaged directional antenna SINR. The index number of the BS with the highest SINR is stored as the label. This process is repeated a number of times, with random BS positions, UAV heights and building deployments, to populate our datasets. Having generated the two datasets we train our NN model to infer through the chosen features which BS the UAV should associate with, for a given set of environmental parameters.

\section{NumericAl RESUlts}

In this section we evaluate the performance of our NNbased BS association. We achieve this by simulating the urban environment over a number of Monte Carlo (MC) trials where the BS distributions and the building heights are random, and recording the probability of the UAV having coverage after choosing a BS to associate with (referred to as the coverage probability). For comparison, we additionally evaluate the performance of the UAV when it associates with a BS following a simple heuristic scheme. As the UAV has an omni-directional antenna which monitors the BS signal powers, the UAV can adopt an association scheme where it associates with the BS which has the strongest SINR, as directly measured by the omni-directional antenna. The UAV also knows the locations of the nearby BSs, therefore it can adopt an association scheme wherein it chooses to associate with the BS that has the closest horizontal distance to it, irrespective of the received signal power at the omni-directional antenna. For both association schemes, the UAV makes a decision based on the information immediately available to it, and aligns the directional antenna towards its chosen associated BS. Table 1 gives the values of
TABLE I

NUMERICAL RESUlt PARAMETERS

\begin{tabular}{|c|c|}
\hline Parameter & Value \\
\hline Carrier Frequency & $2 \mathrm{GHz}$ \\
Building density & $300 / \mathrm{km}^{2}$ \\
Building land coverage & 0.5 \\
Building height scale parameter & $20 \mathrm{~m}$ \\
LoS pathloss exponent $\alpha_{L}$ & 2.1 \\
NLoS pathloss exponent $\alpha_{N}$ & 4 \\
LoS multipath fading parameter $m_{L}$ & 1 \\
NLoS multipath fading parameter $m_{N}$ & 1 \\
BS transmit power $p$ & $40 \mathrm{~W}$ \\
Near-field pathloss $c$ & $-38.4 \mathrm{~dB}[17]$ \\
SINR threshold $\theta$ & $0 \mathrm{~dB}$ \\
Noise power $\sigma^{2}$ & $8 \cdot 10^{-13} \mathrm{~W}[17]$ \\
BS height above ground $\gamma_{G}$ & $30 \mathrm{~m}$ \\
Number of BS antenna elements $N_{t}$ & 8 \\
Candidate BS number $\zeta$ & 10 \\
Interfering BS number $\xi$ & 20 \\
\hline
\end{tabular}

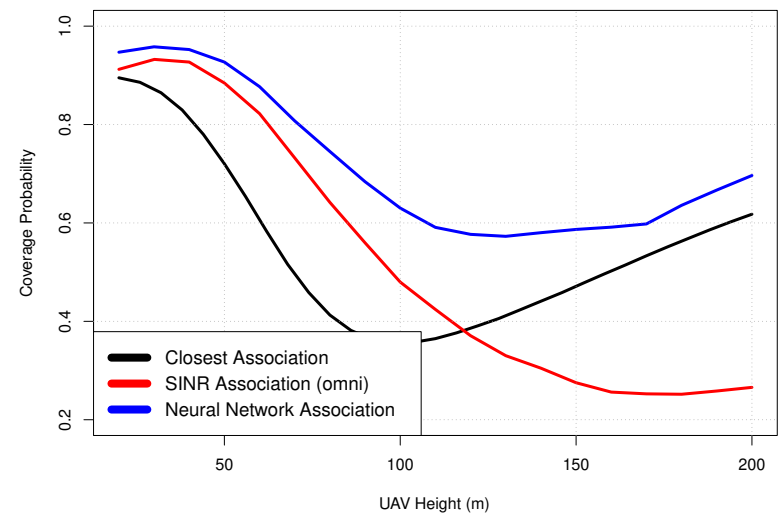

Fig. 3. Coverage probability of the UAV as a function of the height $\gamma$, given beamwidth $\omega$ of 45 degrees and a BS density $\lambda$ of $5 / \mathrm{km}^{2}$. The blue line denotes the performance under our NN association approach, the black line denotes the mathematically-derived performance for closest-BS association derived in [5] and [6], and the red line denotes strongest SINR association as measured from the omni-directional antenna.

the environmental parameters. In figs. 3 to 5 the results for the closest-BS association are obtained via the mathematical expressions derived by us in our prior works [5] and [6], while the rest are found via simulations.

The results in fig. 3 show that the $\mathrm{NN}$ association strategy gives a consistent improvement to the coverage probability when compared to the non-NN closest-BS or strongest SINR association, at all values of the height of the UAV. At low heights the NN strategy gives very similar performance to the strongest SINR association, as interference effects are less of an issue, and therefore choosing the BS with the strongest SINR observed by the omni-directional antenna appears to be the optimum strategy. At large UAV heights, due to BS antenna downtilt the stronger SINR measured by the omni-directional antenna will come from more distant BSs, which will result in more interference for the directional antenna if the UAV associates with one of them (due to a shallower tilt angle and greater area $\mathcal{W}$ ). As a result, at large heights the UAV must prioritise connecting to a closer BS, even if this will result in a 


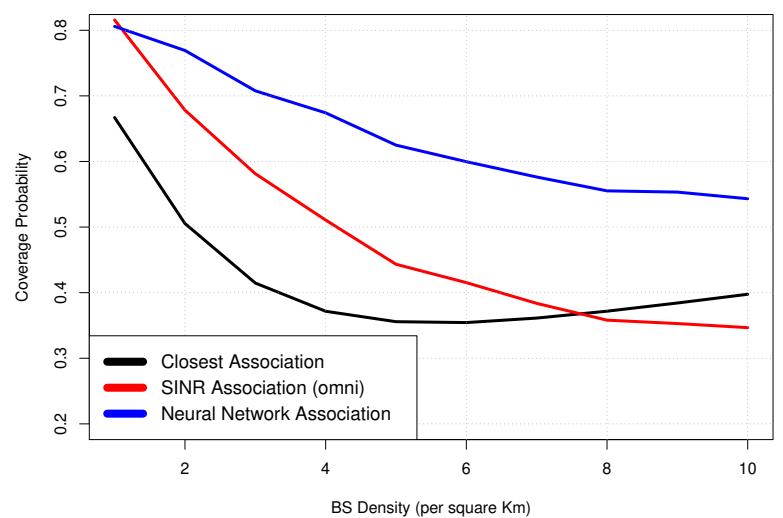

Fig. 4. Coverage probability of the UAV as a function of the BS density $\lambda$, given beamwidth $\omega$ of 45 degrees and a UAV height $\gamma$ of $100 \mathrm{~m}$. The blue line denotes the performance under our NN association approach, the black line denotes the mathematically-derived performance for closest-BS association derived in [5] and [6], and the red line denotes strongest SINR association as measured from the omni-directional antenna.

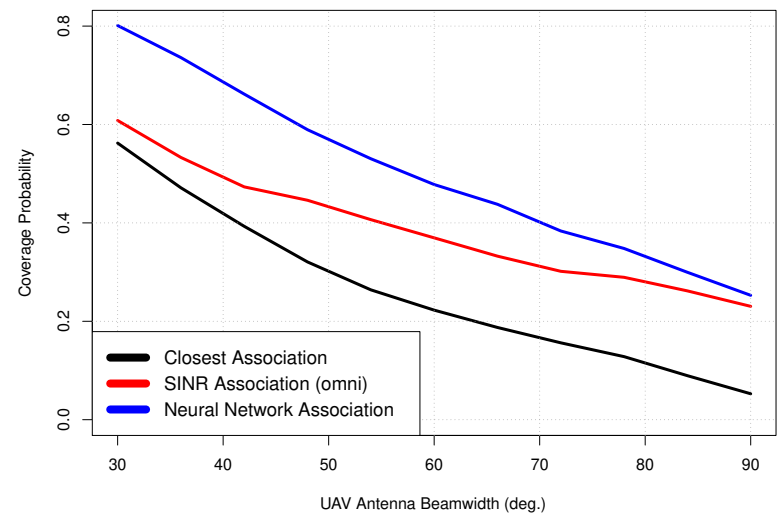

Fig. 5. Coverage probability of the UAV as a function of the UAV antenna beamwidth $\omega$, given BS density $\lambda$ of $5 / \mathrm{km}^{2}$ and a UAV height of $100 \mathrm{~m}$. The blue line denotes the performance under our NN association approach, the black line denotes the mathematically-derived performance for closest-BS association derived in [5] and [6], and the red line denotes strongest SINR association as measured from the omni-directional antenna.

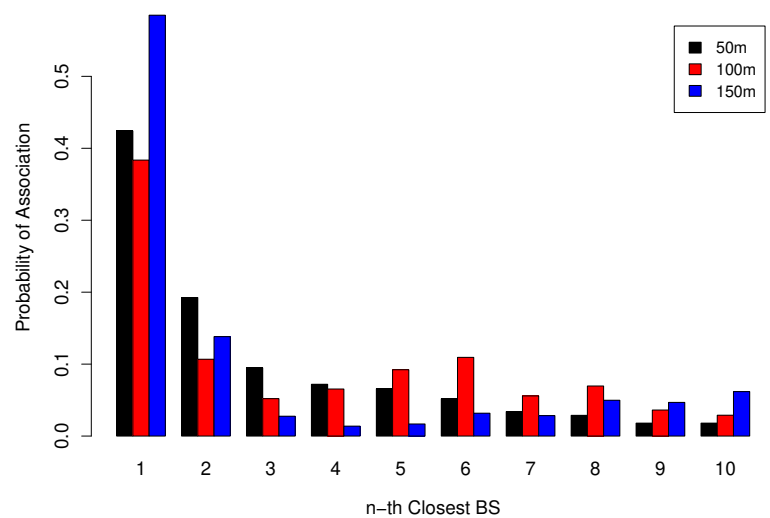

Fig. 6. Probability of the NN choosing to associate to the $n$-th closest BS, given a certain UAV height. lower BS antenna gain, due to a bigger misalignment between the BS antenna tilt, and the UAV-BS vertical angle. The NN recognises this, and so allows the UAV to massively improve its coverage probability over the strongest SINR association case. The biggest $\mathrm{NN}$ gains are achieved in the middle range of heights, where both the closest and strongest SINR association strategies give poor performance. This is due to the fact that at these heights, on one hand the UAV has unobstructed LoS channels to distant interfering BSs, while on the other hand the UAV is not so high up that it can mitigate interference through tilting its antenna down. The $\mathrm{NN}$ is able to reduce the performance loss at these heights by choosing a BS which offers a good tradeoff between a high sidelobe antenna gain, low signal pathloss, as well as low interference.

The results in fig. 4 show that the overall network performance deteriorates as the BS density increases, due to increasing interference. At lower densities better performance is achieved by associating to the BS with the strongest measured SINR, while at larger densities connecting to the closest BS gives better results. The NN is able to outperform both association strategies, with a bigger performance improvement observed for the higher BS densities where the UAV experiences more interference.

In fig. 5 we show the impact of the UAV directional antenna beamwidth $\omega$ on the coverage probability. Increasing the beamwidth causes performance to deteriorate for all association policies, due to the resulting increase in interference observed by the UAV. Note that as the beamwidth increases the peformance gain offered by our NN solution decreases compared to the strongest association, as at larger beamwidths the intelligence of the $\mathrm{NN}$ is not sufficient to mitigate the impact of interference.

The plot in fig. 6 shows the probability of the NN choosing a certain BS to associate with, for different UAV heights. We can see that the UAV will be served by the closest BS approximately half of the time for the tested heights, due to the impact of antenna misalignment when the UAV and the BS are a short horizontal distance apart. The NN will instead sometimes prefer to connect to BSs further away, with the shape of the BS sidelobes having a noticeable impact on the probability distribution of the chosen BS. Consider, for example, how the NN will very rarely choose the third-closest BS when the UAV is at 100 meters; the NN has learned during training that the fourth, fifth and sixth BSs are more likely to give a better SINR, even if the distance-dependent pathloss and the interference is greater.

\section{CONCLUSION}

In this paper we have proposed an NN-based association policy that allows a UAV to choose a suitable BS to connect to, based on information about nearby BS transmit powers, their distances to the UAV, as well as the locations of nearby BSs which may cause interference. We demonstrated that the resulting $\mathrm{NN}$ was able to increase the probability of UAV coverage significantly, compared to typical non-NN BS selection schemes. 
In our future work we plan to extend the work in this paper by considering a scenario where the UAV moves through the urban environment, while making BS association decisions. This will introduce handovers to the UAV association problem, which will complicate the decision process. We intend to apply machine learning to balance the UAV channel quality requirements with the additional mobility management requirements.

\section{ACKNOWLEDGEMENT}

This material is based upon works supported by the Science Foundation Ireland under Grants No. 17/NSFC/5224 and 13/RC/2077.

\section{REFERENCES}

[1] X. Ge, "Ultra-Reliable Low-Latency Communications in Autonomous Vehicular Networks," IEEE Transactions on Vehicular Technology, vol. 68, no. 5, pp. 5005-5016, 2019.

[2] M. Mozaffari et al., "A Tutorial on UAVs for Wireless Networks: Applications, Challenges, and Open Problems," IEEE Communications Surveys \& Tutorials, vol. 21, no. 3, pp. 2334-2360, Third Quarter 2019

[3] X. Lin et al., "The Sky Is Not the Limit: LTE for Unmanned Aerial Vehicles," IEEE Communications Magazine, vol. 56, no. 4, pp. 204 210, April 2018.

[4] "TR 36.777: Technical Specification Group Radio Access Network; Study on Enhanced LTE Support for Aerial Vehicles (Release 15)," 3GPP, Tech. Rep., 2018.

[5] B. Galkin, J. Kibilda, and L. A. DaSilva, "Backhaul for Low-Altitude UAVs in Urban Environments," in IEEE International Conference on Communications (ICC), May 2018.

[6] R. Amer et al., "Performance Analysis of Mobile Cellular-Connected Drones under Practical Antenna Configurations," in IEEE International Conference on Communications (ICC), June 2020.

[7] M. M. Azari, F. Rosas, and S. Pollin, "Reshaping Cellular Networks for the Sky: The Major Factors and Feasibility," in IEEE International Conference on Communications (ICC), May 2018.

[8] — "Cellular Connectivity for UAVs: Network Modeling, Performance Analysis and Design Guidelines," IEEE Transactions on Wireless Communications, vol. 18, no. 7, pp. 3366-3381, July 2019.

[9] G. Geraci et al., "Supporting UAV Cellular Communications through Massive MIMO," IEEE International Conference on Communications Workshops (ICC Workshops), May 2018.

[10] M. Chen et al., "Artificial Neural Networks-Based Machine Learning for Wireless Networks: A Tutorial," IEEE Communications Surveys Tutorials, vol. 21, no. 4, pp. 3039-3071, Fourth Quarter 2019.

[11] S. Chen et al., "Learning-Based Remote Channel Inference: Feasibility Analysis and Case Study," IEEE Transactions on Wireless Communications, vol. 18, no. 7, pp. 3554-3568, July 2019.

[12] M. Arvinte, M. Tavares, and D. Samardzija, "Beam Management in 5G NR using Geolocation Side Information," in 2019 53rd Annual Conference on Information Sciences and Systems (CISS), March 2019.

[13] A. Alkhateeb et al., "Deep Learning Coordinated Beamforming for Highly-Mobile Millimeter Wave Systems," IEEE Access, vol. 6, no. June, pp. 37 328-37348, 2018.

[14] "Recommendation P.1410-5 "Propagation Data and Prediction Methods Required for the Design of Terrestrial Broadband Radio Access Systems Operating in a Frequency Range From 3 to $60 \mathrm{GHz}$,', ITU-R, Tech. Rep., 2012.

[15] X. Xu and Y. Zeng, "Cellular-Connected UAV: Performance Analysis with 3D Antenna Modelling," in IEEE International Conference on Communications Workshops (ICC Workshops), May 2019.

[16] "R Interface to Keras." [Online]. Available: https://keras.rstudio.com/

[17] H. Elshaer et al., "Downlink and Uplink Cell Association With Traditional Macrocells and Millimeter Wave Small Cells," IEEE Transactions on Wireless Communications, vol. 15, no. 9, pp. 6244-6258, Sept. 2016. 